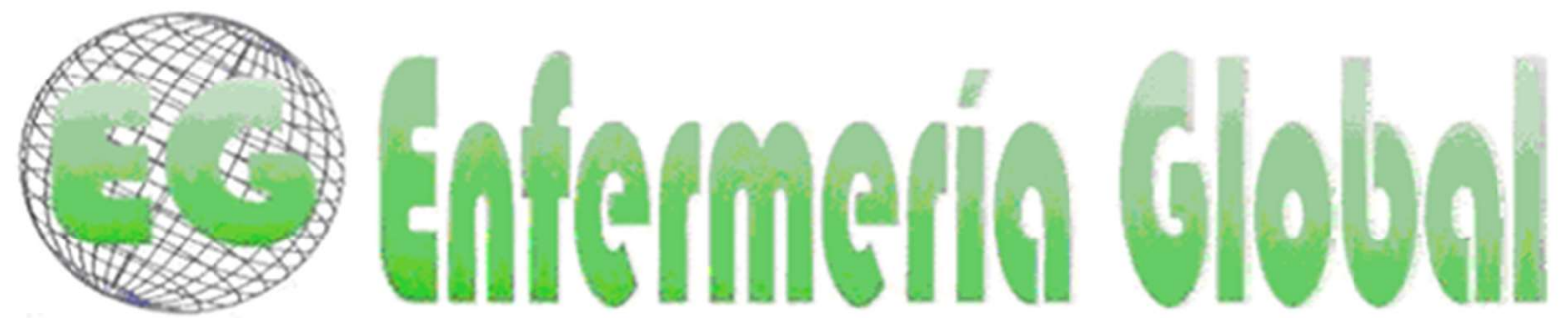

\title{
ORIGINALES
}

\section{Creencias y prácticas alimentarias en embarazo y puerperio: aplicación del Modelo de Tradiciones de Salud}

Beliefs and dietary practices in pregnancy and the puerperium: application of the Health Traditions Model

\author{
Elena Andina-Díaz ${ }^{1}$ \\ Maria Fátima Silva Vieira Martins ${ }^{2}$ \\ José Siles-González ${ }^{3}$
}

1 Facultad Ciencias de la Salud. Campus Vegazana. Universidad de León. Grupo de Investigación SALBIS. Universidad de León. Grupo de Investigación EYCC. Universidad de Alicante. España. elena.andina@unileon.es

2 Escuela Superior de Enfermería. Campus de Gualtar. Universidad del Minho. Braga. Portugal.

${ }^{3}$ Facultad Ciencias de la Salud. Universidad de Alicante. Grupo de Investigación EYCC. Universidad de Alicante. España.

\section{https://doi.org/10.6018/eglobal.413651}

Recibido: 6/02/2020

Aceptado: 22/03/2020

\section{RESUMEN:}

Objetivo: Describir las creencias y prácticas culturales relacionadas con la alimentación durante el embarazo y puerperio en mujeres adultas (mayores de 60 años) en dos culturas diferentes, aplicando el Modelo de Tradiciones de Salud.

Método: Se llevó a cabo un estudio cualitativo. Participaron 16 mujeres residentes durante su embarazo/parto/puerperio en un área rural de Braga (Portugal), o León (España). La técnica de recogida de información fue la entrevista semiestructurada. Se hizo un análisis de contenido, siguiendo el Modelo de Tradiciones de Salud.

Resultados: Se identificaron creencias y prácticas relacionadas con la alimentación, encaminadas a proteger, mantener y recuperar la salud de la madre/recién nacido, desde la esfera física/mental/espiritual (9 dimensiones interrelacionadas).

Conclusión: Se describieron creencias y prácticas alimentarias en embarazo/puerperio de mujeres mayores, constatando el papel de la cultura en las mismas. Se consideraron 9 dimensiones interrelacionadas, y el rol relevante de familiares/allegadas. Estos datos pueden ayudarnos a planificar acciones de salud maternal en la actualidad, participativas (familia/comunidad), corregir ciertas prácticas, y proporcionar cuidados congruentes con la cultura de las mujeres. Ello puede ayudar a transformar creencias, o valores y actitudes que incardinan una determinada forma cultural en la enfermería.

Palabras clave: Modelo de Enfermería; Cuidados de Enfermería; Cultura; Enfermería Materno-Infantil 


\begin{abstract}
:
Objective: To describe the cultural beliefs and practices related to food during pregnancy and the puerperium in adult women (over 60 years old) in two different cultures by applying the Health Traditions Model.

Method: A qualitative study was carried out with the participation of 16 women resident during their pregnancy / childbirth / puerperium in a rural area of Braga (Portugal), and León (Spain). The information collection technique was the semi-structured interview. A content analysis was made, following the Health Traditions Model.

Results: Beliefs and dietary practices related to feeding were identified, aimed at protecting, maintaining and recovering the health of the mother / newborn, from the physical / mental / spiritual sphere (9 interrelated dimensions).

Conclusion: Eating beliefs and practices in pregnancy / puerperium of older women were described, confirming the role of culture in them. 9 interrelated dimensions were considered, as well as the relevant role of family / relatives. These data can help us plan for current, participatory (family / community) maternal health actions, correct certain practices, and provide care consistent with the culture of women. This can help transform beliefs, or values and attitudes that embody a certain cultural form in nursing.
\end{abstract}

Key words: Nursing Model; Nursing care; Culture; Maternal and Child Nursing

\title{
INTRODUCCIÓN
}

La Atención Primaria, como parte integrante del sistema de salud, es un conjunto de atenciones globales, por medio de las cuales la población puede participar activamente en su propio proceso de salud para adquirir nuevos conocimientos. Si la entendemos dentro del contexto del paradigma sociocrítico, los profesionales de salud se consideran agentes de cambio sociosanitario, en cuyo proceso implican al individuo y la comunidad(1). En este marco, la mujer embarazada y su familia participan en dicha acción hacia el cambio, y las atenciones, personalizadas, les permitirán concebir esa etapa de forma positiva ${ }^{(2)}$.

El cuidado de salud permite determinar una relación social y humana entre la matrona y la embarazada, que requiere conocer su historia de vida social, familiar y cultural. En ese sentido, la atención cultural se entiende como el conjunto de valores, creencias y expresiones estructuradas y conocidas de forma cognitiva que ayudan, apoyan, facilitan o potencian a las personas o grupos para que mantengan su salud o bienestar, mejoren su situación o modo de vida, prevengan enfermedades o se ocupen de la discapacidad o la muerte ${ }^{(3)}$.

El embarazo y puerperio integra una etapa significativa en la vida de la mujer y su familia. En la mayoría de las sociedades, es un vínculo con la diversidad cultural de cada generación ${ }^{(4)}$, y la salud y bienestar de las mujeres entonces dependerá del contexto cultural en el que vivan. Las embarazadas suelen poseer conocimientos culturales basados en historias de vida y experiencias sociales transmitidas de mujer a mujer ${ }^{(5)}$, por lo que las enseñanzas de los ancestros son incorporadas y consideradas importantes, debido a la experiencia y al conocimiento compartido ${ }^{(6-8)}$. Diversos estudios muestran cómo el rol de las mujeres (madres/abuelas) tiene relevancia, por ejemplo, a la hora de tomar decisiones de $\operatorname{salud}^{(9,10)}$.

Esta etapa está llena de transformaciones físicas, psicológicas y emocionales, con sus correspondientes cuidados, llenos de restricciones/prohibiciones. Los conocimientos/prácticas adquiridas, que juegan un papel importante para la identidad de grupo y para la supervivencia, se pueden convertir en obstáculos, existiendo 
creencias, actitudes y comportamientos que pueden influir negativamente en la salud $(2,3,11,12)$.

La alimentación durante el ciclo grávido-puerperal está impregnado, además de la dimensión nutricional, de la cultural(13). Encontramos ejemplos de investigaciones en las que se muestran creencias tradicionales sobre alimentos nocivos/beneficiosos para la mujer durante el embarazo(14), así como creencias/normas socioculturales relativas al acto de comer, beneficiosas para la madre y su hijo ${ }^{(15)}$.

Por todo ello, pensamos que identificar creencias y prácticas culturales de nuestras antepasadas, relativas a los cuidados maternales relacionados con la alimentación, nos puede ayudar a entender claves para planificar en la actualidad acciones de salud participativas (considerando mujer/familia/comunidad), corregir prácticas, y proporcionar cuidados considerando las creencias culturales de las mujeres.

Para responder a esa pregunta, diversos investigadores enfermeros han creado modelos y teorías, con aplicaciones interesantes en la práctica enfermera ${ }^{(11,16-19)}$. El fin ha sido poder profundizar en las aportaciones de la cultura tiene en la esfera de la salud. Entre ellos, rescatamos el Modelo de Tradiciones de Salud. Este modelo, creado por Spector ${ }^{(11)}$, explica desde una perspectiva tradicional, cómo las personas de la mayoría de las culturas, buscan el equilibrio con la familia, comunidad y fuerzas de la naturaleza, creando pautas culturales relacionadas con la prevención, protección, mantenimiento y recuperación de su salud a nivel físico, mental y espiritual (nueve dimensiones interrelacionadas). Aplicar este modelo a los cuidados de alimentación en la etapa de embarazo y puerperio, en diferentes culturas, puede ayudarnos a constatar cómo la cultura interviene a la hora de configurar creencias y prácticas relativas a la alimentación, y las dimensiones implicadas.

Con base a estas consideraciones, el objetivo general de este estudio fue: describir las creencias y prácticas culturales relacionadas con la alimentación durante el embarazo y el puerperio en mujeres adultas (mayores de 60 años) ${ }^{20}$ en dos culturas diferentes, aplicando el Modelo de Tradiciones de Salud.

\section{MÉTODO}

Dado que el objeto de la investigación fue describir las creencias y prácticas relacionadas con la alimentación durante el embarazo y puerperio, se utilizó metodología cualitativa.

El emplazamiento (áreas rurales del Concelho de Amares (Braga, Portugal), y Ayuntamientos de Almanza y Cebanico (León, España)) y las participantes/datos (seleccionadas mediante un muestreo de conveniencia) fueron tomados de dos investigaciones ya realizadas ${ }^{(2,12)}$, por contar con aspectos comunes para poder comparar, y que pasamos a detallar.

Los criterios de selección aplicados en ambos estudios habían sido: mujeres mayores de 60 años, nativas (una de las áreas), residentes allí durante embarazo/parto/puerperio, capaces de aportar información relevante. Los motivos de exclusión: no desear participar en el estudio, dificultades para comunicarse. 
Dos de las investigadoras, personas conocidas allí, habían sido las encargadas de acceder al área y recoger la información (rapport). En cada área, se seleccionó a mujeres (cumplimiento de criterios), invitándoles a participar. Mediante la técnica de bola de nieve, se accedió al resto. Todas las mujeres invitadas participaron finalmente. Se recogió información hasta llegar a la saturación. La muestra final estuvo constituida por 16 mujeres.

La técnica de recogida de información fue la entrevista semiestructurada. Los temas abordados en las entrevistas formaron parte de dos estudios mayores ${ }^{(2,12)}$, tomando para este trabajo las respuestas relacionadas con el objeto de estudio. Las entrevistas se realizaron en casa de las participantes (45-60 minutos cada una). Se utilizó grabadora de voz. Posteriormente se contactó con las participantes para confirmar los datos.

En cuanto al análisis de datos, ya específico realizado para el estudio que ahora se presenta, se realizó trascripción literal de las entrevistas y análisis manual de contenido de las dos áreas por separado, siguiendo propuesta de Bardin(21): i) preanálisis, ii) exploración de material y iii) tratamientos de los resultados por inferencia e interpretación. Posteriormente, se llevó a cabo la puesta en común. Para organizar la información se utilizó el Modelo de Tradiciones de Salud ${ }^{(11)}$, utilizando las dimensiones del mismo a modo de categorías.

Sobre los aspectos éticos, cada participante firmó un consentimiento informado. Se siguieron las recomendaciones de la Declaración de Helsinki y Convención de Oviedo. Se obtuvo la aprobación del Comité de Ética (UA-2015-08-18).

\section{RESULTADOS}

Las participantes del estudio fueron 16 mujeres. En la Tabla 1 aparecen detalladas las características sociodemográficas de las mismas.

Tabla 1. Características sociodemográficas de las participantes en el estudio

\begin{tabular}{|c|c|c|c|c|c|c|c|}
\hline Entrevistada & Edad & Estudios & Profesión & $\begin{array}{c}N^{\circ} \\
\text { de } \\
\text { hijos }\end{array}$ & Lugar parto & $\begin{array}{c}\text { Estado } \\
\text { civil }\end{array}$ & $\begin{array}{c}\text { Área } \\
\text { geográfica }\end{array}$ \\
\hline E1e & $\begin{array}{c}80 \\
\text { años }\end{array}$ & Primarios & Ama casa & 3 & Hospital & Casada & $\begin{array}{c}\text { Almanza/Cb } \\
\text { (ES) }\end{array}$ \\
\hline E2e & $\begin{array}{c}72 \\
\text { años }\end{array}$ & Primarios & Ama casa & 5 & Hogar/Hospital & Viuda & $\begin{array}{c}\text { Almanza/Cb } \\
(\mathrm{ES})\end{array}$ \\
\hline E3e & $\begin{array}{c}82 \\
\text { años }\end{array}$ & Primarios & Ama casa & 3 & Hogar/Hospital & Viuda & $\begin{array}{c}\text { Almanza/Cb } \\
(\mathrm{ES})\end{array}$ \\
\hline E4e & $\begin{array}{c}84 \\
\text { años }\end{array}$ & Primarios & Ama casa & 5 & Hogar & Viuda & $\begin{array}{c}\text { Almanza/Cb } \\
\text { (ES) }\end{array}$ \\
\hline E5e & $\begin{array}{c}86 \\
\text { años }\end{array}$ & Primarios & Ama casa & 5 & Hogar/Hospital & Viuda & $\begin{array}{c}\text { Almanza/Cb } \\
(\mathrm{ES})\end{array}$ \\
\hline E6e & $\begin{array}{c}82 \\
\text { años }\end{array}$ & Primarios & Ama casa & 2 & Hogar & Viuda & $\begin{array}{c}\text { Almanza/Cb } \\
(\mathrm{ES})\end{array}$ \\
\hline E7e & $\begin{array}{c}80 \\
\text { años }\end{array}$ & Primarios & Ama casa & 17 & Hogar & Casada & $\begin{array}{c}\text { Almanza/Cb } \\
\text { (ES) }\end{array}$ \\
\hline
\end{tabular}




\begin{tabular}{|c|c|c|c|c|c|c|c|}
\hline E8e & $\begin{array}{c}94 \\
\text { años }\end{array}$ & Primarios & Ama casa & 5 & Hogar & Viuda & $\begin{array}{c}\text { Almanza/Cb } \\
\text { (ES) }\end{array}$ \\
\hline E1p & $\begin{array}{c}85 \\
\text { años }\end{array}$ & Primarios & Ama casa & 5 & Hogar & Viuda & $\begin{array}{c}\text { Amares } \\
\text { (PO) }\end{array}$ \\
\hline$E 2 p$ & $\begin{array}{c}86 \\
\text { años }\end{array}$ & Primarios & $\begin{array}{c}\text { Ama } \\
\text { casa/parteira }\end{array}$ & 3 & Hogar & Viuda & $\begin{array}{c}\text { Amares } \\
(\mathrm{PO})\end{array}$ \\
\hline E3p & $\begin{array}{c}60 \\
\text { años }\end{array}$ & Primarios & Ama casa & 4 & Hogar & Viuda & $\begin{array}{c}\text { Amares } \\
\text { (PO) }\end{array}$ \\
\hline$E 4 p$ & $\begin{array}{c}80 \\
\text { años }\end{array}$ & $\begin{array}{c}\text { No sabe } \\
\text { leer/escribir }\end{array}$ & Ama casa & 4 & Hogar & Viuda & $\begin{array}{c}\text { Amares } \\
\text { (PO) }\end{array}$ \\
\hline$E 5 p$ & $\begin{array}{c}64 \\
\text { años }\end{array}$ & $\begin{array}{c}\text { No sabe } \\
\text { leer/escribir }\end{array}$ & Ama casa & 3 & Hogar & Viuda & $\begin{array}{c}\text { Amares } \\
(\mathrm{PO})\end{array}$ \\
\hline E6p & $\begin{array}{c}74 \\
\text { años }\end{array}$ & Primarios & Ama casa & 7 & Hogar & Viuda & $\begin{array}{c}\text { Amares } \\
\text { (PO) }\end{array}$ \\
\hline E7p & $\begin{array}{c}62 \\
\text { años }\end{array}$ & Primarios & Ama casa & 4 & Hospital & Casada & $\begin{array}{c}\text { Amares } \\
(\mathrm{PO})\end{array}$ \\
\hline$E 8 p$ & $\begin{array}{c}65 \\
\text { años }\end{array}$ & Primarios & Ama casa & 4 & Hogar & Viuda & $\begin{array}{c}\text { Amares } \\
\text { (PO) }\end{array}$ \\
\hline
\end{tabular}

*ES: ESpaña / PO:: Portugal

Los resultados obtenidos fueron clasificados en 3 categorías principales, y 9 interrelacionadas, correspondientes a las dimensiones descritas en el Modelo de Tradiciones de Salud (Tabla 2).

Tabla 2. Aplicación del Modelo de Tradiciones de Salud, nueve facetas interrelacionadas de Salud y métodos comunitarios y personales de mantener, proteger y restaurar la salud, en el embarazo y puerperio

\begin{tabular}{|c|c|c|c|}
\hline $\begin{array}{l}\text { EMBARAZO I } \\
\text { PUERPERIO }\end{array}$ & Física & Mental & Espiritual \\
\hline \multirow{4}{*}{ 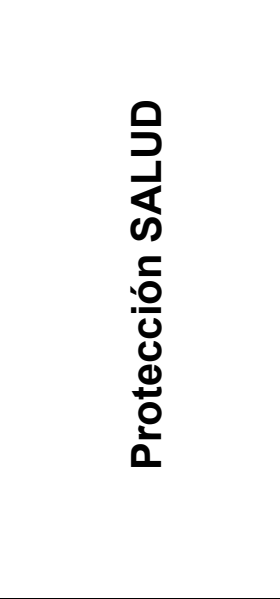 } & $\begin{array}{l}\text { Promoción calidad } \\
\text { amamantamiento }\end{array}$ & $\rightarrow \rightarrow$ & $\rightarrow \rightarrow$ \\
\hline & $\begin{array}{c}\text { Prevención de } \\
\text { cólicos recién } \\
\text { nacido }\end{array}$ & $\rightarrow \rightarrow$ & $\rightarrow \rightarrow$ \\
\hline & $\leftarrow \leftarrow$ & $\leftarrow \leftarrow$ & $\begin{array}{c}\text { Antojos o deseos } \\
\text { embarazada }\end{array}$ \\
\hline & $\leftarrow \leftarrow$ & $\leftarrow \leftarrow$ & $\begin{array}{l}\text { Prevención mal de ojo } \\
\text { recién nacido }\end{array}$ \\
\hline \multirow{2}{*}{ 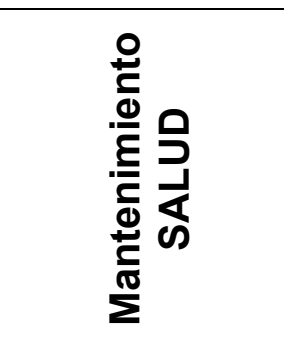 } & $\begin{array}{c}\text { Alimentación recién } \\
\text { nacido primeras } \\
\text { horas }\end{array}$ & $\rightarrow \rightarrow$ & $\rightarrow \rightarrow$ \\
\hline & $\begin{array}{l}\text { Alimentación en } \\
\text { caso de que } \\
\text { lactancia materna } \\
\text { no fuera posible }\end{array}$ & $\rightarrow \rightarrow$ & $\rightarrow$ \\
\hline
\end{tabular}




\begin{tabular}{|c|c|c|c|}
\hline \multirow{3}{*}{ 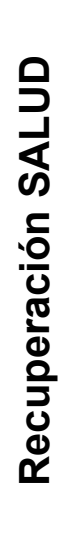 } & $\begin{array}{c}\text { Alimentación } \\
\text { puérpera }\end{array}$ & $\rightarrow \rightarrow$ & $\rightarrow \rightarrow$ \\
\hline & $\leftarrow \leftarrow$ & $\leftarrow \leftarrow$ & $\begin{array}{c}\text { Rituales relativos a } \\
\text { elaboración de } \\
\text { comidas para } \\
\text { parturienta }\end{array}$ \\
\hline & $\leftarrow$ & $\leftarrow \leftarrow$ & $\begin{array}{l}\text { Visitas posparto, } \\
\text { alimentos }\end{array}$ \\
\hline
\end{tabular}

En cuanto a creencias y prácticas relacionadas con alimentación relativas a proteger la salud de madre o recién nacido, a nivel físico, la primera a la que hicieron mención estaba relacionada con la promoción de la calidad del amamantamiento. Las participantes comentaron cómo determinados alimentos ayudaban a la producción de leche:

Comer demasiado bacalao y demasiadas sardinas es muy bueno... si comía un poco de bacalao, esa noche tenía más leche (E4p).

El caldo era ligero... para la leche era mejor (E4e).

Así mismo, mencionaron bebidas como el vino:

Por la noche la partera (mi tía, hermana de mi madre) me dejaba un vasín de vino dulce, y 3 o 4 galletas para cuando diera de mamar al niño (E8e).

En cuanto a la lactancia, dicen que es muy bueno beber un poco de vino con azúcar y una corteza de pan caliente en su interior (E1p).

Se mencionaron también conductas perjudiciales:

Si una mujer que está amamantando tira sus sobras de comida a la basura y una mujer embarazada se las come, la madre se quedará sin leche (E5p).

Las sobras podrían ser consumidas por animales que están preñados y entonces nuestra leche se secaría (E2p).

La segunda costumbre popular recogida estaba relacionada con la prevención de cólicos en el recién nacido:

No podía comer sopa de col con frijoles porque le pasaba al bebé y podía hacerle daño, cólico (E1p).

En las esferas mental y espiritual, recogimos creencias relacionadas con antojos o deseos alimenticios durante el embarazo que debían satisfacerse para evitar daños en el bebé. Su cumplimiento proporcionaba tranquilidad y bienestar en la embarazada a nivel mental y espiritual ( $\mathrm{y}$, además bienestar físico). 
Si veía una cosa que me gustaba, si no llenaba la barriga no quedaba a gusto... si no se cumplía salía una mancha... al niño (E2e).

Si una mujer embarazada no ha satisfecho sus deseos alimenticios, comiendo como le plazca, se arriesga a que su hijo nazca con la boca abierta y el cabello perforado $(\mathrm{E} 1 \mathrm{p})$.

Algunos alimentos deseados o beneficiosos mencionados fueron las sardinas, frutas, o bebidas:

Mi suegra solía decirme, tomar un trago (de vino) para tener un niño guapo (E4p).

Beber brandy con el estómago vacío durante toda la gestación con el propósito de "matar al animal", permite que el feto no nazca en forma de animal (E5p).

Por otra parte, se mencionaron alimentos prohibidos, como las naranjas (para evitar niño con piel de naranja (E6p)), las patas de pollo (porque el bebé puede nacer con seis dedos (E1p)), o el pescado:

No se pueden comer peces... los bebés pueden nacer... con la forma o apariencia del animal o con los ojos pequeños... o con manchas... aborto (E4p).

También se recogió una práctica relacionada con el mal de ojo:

Mi suegra echó agua en su pequeña boca para prevenir el mal de ojo (E8p).

En relación al mantenimiento de la salud, la primera práctica recogida relacionada con salud física hacía mención a la alimentación del recién nacido durante las primeras horas. Algunas de las participantes recordaban que el calostro no se consideraba nutritivo, y los 2-3 primeros días, se le alimentaba con sucedáneos como:

El primer día no había leche... (mi madre me daba) un poco de manzanilla... tila o manzanilla... con un poco de azúcar (E3e).

Otra práctica hacía mención a la alimentación en caso de que la lactancia materna no fuera posible:

Leche (vaca) rebajada con harina tostada... patatas aplastadas con una yema de huevo (E8e).

Sobre creencias y prácticas relacionadas con la recuperación de la salud a nivel físico de la puérpera, la alimentación de los primeros días estaba basada en alimentos como:

Comía pues caldo de gallina... echaba unos fideos (mi madre, cuando lo preparaba), un poco de arroz o cosas así... me reservaba de comer un poco de las muchas sustancias, dos días (E7e).

Debes comer sopa de pollo después del parto porque es buena para el vientre de la madre (E8p). 
En cuanto a salud mental y espiritual, algunas participantes mencionaron que cuando te ponías de parto, la familia se ocupaba de matar la gallina y preparaba el caldo, lo que les ayudaba a recuperarse:

Vino una cuñada, y dijo, voy a hacerte el caldo de gallina que me sale muy bueno (E2e).

Mi tía había matado ya una gallina porque ya había estado yo algo (revuelta) (E5e).

La parturienta recibía la visita de vecinas, mostrando su apoyo, que repercutía en su bienestar:

Después nos iban a ver... las vecinas, (llevaban) chocolate, o media docena de huevos, la visita (E6e).

\section{DISCUSIÓN}

Si comparamos algunas de las creencias y prácticas identificadas aquí con las existentes en otras comunidades, pudimos establecer igualmente ciertas similitudes. Al respecto, por ejemplo, en la esfera de la protección de la salud, a nivel físico, las participantes hicieron mención a creencias y prácticas relativas a promocionar la calidad del amamantamiento. La leche materna es el alimento ideal para el bebé debido a sus propiedades nutricionales e inmunológicas, y se considera por ello una práctica fundamental para promover, proteger y apoyar su salud. En ese sentido, la preocupación por la producción de leche materna (calidad y cantidad), la encontramos reflejada igualmente en otros estudios llevados a cabo en otras comunidades ${ }^{(4,22)}$.

El vino, bebida mencionada por las mujeres del estudio, ha estado presente de igual manera en otras culturas ${ }^{(23-24)}$, como hábito alimentario. Su consumo ha sido raramente cuestionado, considerado incluso como un tónico fuerte. Las sopas de caballo cansadas que se daban a los niños en Portugal confirman esta idea(4). Sin embargo, se sabe que este repercute negativamente a corto y largo plazo en la salud de los lactantes, y reduce la producción de la leche en la madre ${ }^{(23)}$.

Otra creencia mencionada en nuestro estudio, en este caso relativa a la prevención de cólicos, igualmente la encontramos citada en otros trabajos, como el de Kidd et al. ${ }^{(25)}$, en el que destacan la creencia de las madres de que el cólico de su recién nacido estaba relacionado con el dolor abdominal relacionado con la alimentación, y había que eliminar alimentos de su dieta. Al respecto, autores Miranda et al. ${ }^{(5)}$ advierten que el uso de té en el recién nacido es perjudicial, por la inmadurez de los riñones y el exceso de líquidos (leche materna, tés y agua), pudiendo causar lesiones, así como reducir la succión, estimulación y producción de leche materna.

Encontramos menciones actuales en la literatura(26), que se asemejan a las narradas por nuestras participantes, relativas al cumplimiento de los antojos, y que tenían como fin proteger y proporcionar bienestar físico, mental y espiritual a la futura madre, y del recién nacido. 
Las restricciones dietéticas parece que tienen por objeto proteger al individuo de situaciones en las que su cuerpo está más expuesto a los riesgos de agresión externa y existe una conciencia de necesidad de protegerlo. En esa línea, encontramos en nuestro estudio cómo muchas de las prohibiciones alimentarias del embarazo estaban relacionadas con el temor a provocar cambios en los niños. Jacques ${ }^{(27)}$ define la ley sobre el contagio con la idea de que los que han estado en contacto, se mantendrán en contacto. Los alimentos ingeridos tienen propiedades imaginarias que transformarán el cuerpo de la mujer embarazada y del feto. Por ello, la futura madre debe seleccionar los alimentos en función de sus efectos, con el objetivo de hacer un bebé hermoso y sano(2).

En la esfera de la recuperación de la salud, encontramos también estudios en los que se mencionan restricciones dietéticas y recomendaciones (como alimentos hipercalóricos) parecidas a las mencionadas por las participantes, que tenían como objetivo recuperar la salud a nivel físico de madre y bebé. Por ejemplo, Mao et al.(28) señalaron cómo en el puerperio había restricciones de algunos alimentos y aumento de consumo de otros. Miranda et al. ${ }^{(5)}$ señalaron que comer carne de cerdo, pescado, huevos, repollo o coliflor podían provocar fiebre, infecciones uterinas o inflamaciones; las frutas ácidas, cortar la hemorragia; y la calabaza y la yuca, edema y problemas en los senos. Parece que estas acciones, llenas de símbolos y significados, tenían un doble propósito: expresar la percepción de la salud y la enfermedad y definir o redefinir los roles sociales. En relación a la mencionada y famosa sopa de pollo/caldo de gallina puede deber su éxito al fácil acceso del alimento (económica), ligereza y riqueza de nutrientes ${ }^{(2)}$.

Para finalizar, los alimentos recibidos por las allegadas en el posparto, la visita, mencionados por las participantes del estudio, y que eran considerados en otras culturas como simbólicos ${ }^{(29)}$, formaban parte de un ritual para recuperar las fuerzas tras el parto. La alimentación por ello se volvía requisito básico para promocionar y proteger la salud ${ }^{(30)}$. Por otra parte, esos momentos eran importantes para comprobar el papel de los allegados en el mantenimiento del bienestar familiar. El apoyo (intercambiando bienes y servicios entre individuos), tenía como objetivo mantener o mejorar su bienestar(2), y ayudar a afrontar la nueva situación de ser madre.

Este trabajo no está exento de limitaciones, relacionadas con la ubicación (áreas específicas) y la falta de estudios similares. Sí encontramos en la literatura aplicaciones de otros modelos culturales de salud, si bien no de este. Por ello, como futuras líneas, se podría aplicar este modelo a otras culturas, con el fin de poder establecer comparativas, y compararlo con otros modelos ${ }^{(31,32)}$.

Para finalizar, diremos que se ha cumplido el objetivo del trabajo, describiendo las creencias y prácticas culturales relacionadas con la alimentación durante embarazo y puerperio en mujeres adultas, en dos culturas diferentes, aplicando el modelo de Tradiciones de Salud. Constatamos cómo la cultura intervino a la hora de configurar algunas de sus creencias y prácticas. Estas estaban relacionadas con la prevención, con el mantenimiento y con la recuperación de la salud de la madre o del recién nacido, a nivel físico, mental o espiritual, de manera interrelacionada (9 dimensiones). Tanto la familia como las allegadas (entre ellas sus mayores) jugaron un papel importante a la hora de configurar o mantener algunas de las pautas seguidas, adquiriendo la atención maternal una dimensión comunitaria. Las creencias y 
prácticas identificadas en las zonas objeto de estudio compartieron algunos aspectos esenciales con las de otras áreas más alejadas.

Estos resultados pueden ayudarnos a comprender algunas claves para planificar en la actualidad acciones de salud maternal relativas a alimentación en esta etapa, participativas (en las que considerar a la mujer, pero también a la familia y/o comunidad), corregir ciertas creencias o prácticas perjudiciales, y proporcionar cuidados congruentes con la cultura de las mujeres ${ }^{(6,7,9,10,33)}$, considerando que pueden existir 9 dimensiones relacionadas. Todo ello puede ayudar a transformar creencias, valores y actitudes que incardinan una determinada forma cultural en la enfermería.

\section{CONCLUSIÓN}

A través de la aplicación del Modelo de Tradiciones de Salud se ha podido describir las creencias y prácticas relacionadas con la alimentación durante el embarazo y el puerperio en mujeres adultas (mayores de 60 años) en dos culturas diferentes.

La aplicación de modelos culturales como el Modelo de Tradiciones de Salud, puede ser una herramienta interesante para que los profesionales sanitarios constaten el papel que tiene la cultura a la hora de configurar cuidados relacionados con la salud; descubran prácticas y creencias populares relacionadas con la prevención, mantenimiento y recuperación de salud; y su interrelación con las dimensiones física, mental y espiritual de las personas.

Descubrir creencias y prácticas de nuestros antecesores nos puede ayudar a entender algunas claves para planificar en la actualidad acciones de salud participativas, en las que implicar a familia y comunidad, corregir ciertas prácticas perjudiciales, y proporcionar cuidados congruentes con la cultura de las mujeres. Ello puede ayudar a transformar las creencias, valores y actitudes que incardinan una determinada forma cultural en la enfermería.

\section{REFERENCIAS}

1. Siles-González J. Historia de la enfermería. Madrid: Difusión Avances de Enfermería; 2011.

2. Martins MF. Educação para a Saúde e Vigilância Pré-Natal: Inter-confluências profissionais e familiares. Braga: Instituto de Ciências Sociais da Universidade do Minho; 2011. Doutoramento em Sociologia.

3. Wehbe-Alamah H. Madeleine Leininger's Theory of Culture Care Diversity and Universality. In: Smith MC, Parker ME (ed.). Nursing Theories and Nursing Practice. Philadelphia: FA Davis Company; 2015. p. 303-319.

4. Martins MF. Mitos e Crenças na Gravidez: Sabedoria e segredos tradicionais das mulheres de seis concelhos do Distrito de Braga. Lisboa: Colibri; 2007.

5. Miranda DB, Marostica FC Matão MEL. Influence of cultural factor in the process of care in the postpartum. Revista Eletrônica Gestão \& Saúde. 2015; 6(3):2444-59

6. Sheehy G, Aung Y, Foster AM. "She learned it from her mother and grandmother": Women's experiences with Delivery and post-partum practices in Periurban Yangon, Myanmar. Matern Child Health J. 2016; 20:854-861. doi:10.1007/s10995-016-1918-z 
7. Joseph FI, Earland J. A qualitative exploration of the sociocultural determinants of exclusive breastfeeding practices among rural mothers, North West Nigeria. Int Breastfeed J. 2019; 14:38. doi:10.1186/s13006-019-0231-z

8. Andina-Díaz E, Siles-González J. The domestic participation in birth assistance in the mid-twentieth century. Rev Lat Am Enfermagem. 2016; 24:e2727. doi:10.1590/1518-8345.0574.2727

9. Ngum Chi Watts MC, Liamputtong P, Mcmichael C. Early motherhood: a qualitative study exploring the experiences of African Australian teenage mothers in greater Melbourne, Australia. BMC Public Health. 2015; 10(15):873. doi:10.1186/s12889-0152215-2

10. Herlihy JM, Shaikh A, Mazimba A, Gagne N, Grogan C, et al. Local Perceptions, Cultural Beliefs and Practices That Shape Umbilical Cord Care: A Qualitative Study in Southern Province, Zambia. PLoS ONE. 2013; 8(11): e79191. doi:10.1371/journal.pone.0079191

11. Spector RE. Cultural Competence. Cult Cuid. 2016; 20:44. doi:10.14198/cuid.2016.44.01

12. Andina Díaz E. Valoración sobre la participación de lo doméstico en torno al parto. Creencias y prácticas populares en Almanza y Cebanico (Sahagún, León) durante la primera mitad del Siglo XX. Alicante: Universidad de Alicante; 2015. Doctorado en Enfermería y Cultura de los Cuidados.

13. Moreira LN, Barros DC, Baião MR, Cunha MB. "Quando tem como comer, a gente come": fontes de informações sobre alimentação na gestação e as escolhas alimentares. Physis: Revista de Saúde Coletiva. 2018; 28(3):1-20

14. Arzoaquoi SK, Essuman EE, Gbagbo FY, Tenkorang EY, Soyiri I, Laar AK. Motivations for food prohibitions during pregnancy and their enforcement mechanisms in a rural Ghanaian district. J Ethnobiol Ethnomed. 2015; 11(59):1-9

15. Withers M, Kharazmi N, Lim E. Traditional beliefs and practices in pregnancy, childbirth and postpartum: a review of the evidence from Asian countries. Midwifery. 2018; 158-170. doi:10.1016/j.midw.2017.10.019

16. Fernandes ETBS, Nascimento ER, Ferreira SL, Coelho EAC, Silva LR, Pereira COJ. Cervical cancer prevention among quilombola women in the light of Leininger's theory. Rev Gaúcha Enferm. 2018; 39:e2016-0004. doi:10.1590/19831447.2018.2016-0004

17. Gil-Esteban MD, Solano-Ruiz MC. La aplicación del modelo de competencia cultural en la experiencia del cuidado en profesionales de Enfermería de Atención Primaria. Aten Primaria. 2017; 49(9):549-556. doi:10.1016/j.aprim.2016.10.013

18. Gower S, van der Akker J, Jones M, Dantas JAR. Australian nursing and midwifery educators delivering evidence-based education in Tanzania: a qualitative study. Nurse Educ Pract. 2016; 18:16-22. doi:10.1016/j.nepr.2016.02.009

19. Merritt L. Communicating with Chinese American families in the NICU using the Ginger and Davidhizar transcultural model. Neonatal netw. 2013; 32(5). doi: 10.1891/0730-0832.32.5.335

20. World Health Organization. 2017. [Internet, homepage, may 2017]. 10 facts on ageing and heatlh. World Health Organization. [cited Sep 20, 2019]. Available from: https://www.who.int/features/factfiles/ageing/en/.

21. Bardin L. Analise de Conteúdo. Edição Revista e Atualizada. Lisboa: Edições 70; 2016.

22. Toschi Lahos N, Doumid Borges Pretto A, Alberici Pastore C. Myths and beliefs about breastfeedingin the state of Rio Grande do Sul (Brazil). Mutr Clín Diet Hospi. 2016; 36(4):27-33. doi:10.12873/364toschi 
23. Chien YC, Liu JF, Huang YJ, Hsu CS, Chao JC. Alcohol levels in Chinese lactating mothers after consumption of alcoholic diet during postpartum "doing-the-month" ritual. Alcohol. 2005; 37(3):143-50

24. Straub B, Melvin C, Labbok M. A descriptive study of Cambodian refugee infant feeding practices in the United States. Int Breastfeed J. 2008; 24:3:2. doi:10.1186/1746-4358-3-2

25. Kidd M, Hnatiuk M, Barber J, Woolgar MJ, Mackay MP. "Something is wrong with your milk": qualitative study of maternal dietary restriction and beliefs about infant colic. Can Fam Physician. 2019; 65(3):204-211

26. Blau LE, Lipsky LM, Dempster KW, Eisenberg Colman MH, Siega-Riz AM, Faith MS, Nansel TR. Women's experience and understanding of food cravings in pregnancy : a qualitative study in women receiving prenatal care at the university of North Carolina-Chapel Hill. J Ac Nutr Diet. 2019. doi:10.1016/j.jand.2019.09.020

27. Jacques B. Le festin du foetus: interdits alimentaires et rituels de protection pendant la grossesse», Les Dossiers de I'Obstétrique, Se préparer à l'accouchement, à la naissance, à la parentalité, Editions E.L.P.E.A. 2004: 225-227.

28. Mao LM, Ma LP, Liu N, Chen BH, Lu QG, Ying CJ, Sun XF. Self-reported health problems related to tradicional dietary practices in pospartum women from urban, suburban and rural areas of Hubei province, China: the "zuo yuezi". Asia Pac J Clin Nutr. 2016; 25(1):158-164. doi: 10.6133/apjcn.2016.25.2.03

29. Gélis J. La sage-femme ou le médecin. Une nouvelle conception de la vie. Paris: Fayard; 2004.

30. Marques C. Alimentação, saúde e cultura: um estudo das práticas alimentares em uma comunidade remanescente de quilombo na Amazônia brasileira. Dissertação apresentada ao Programa de Pós graduação em Saúde Pública da Escola Nacional de Saúde Pública Sérgio Arouca, da Fundação Oswaldo Cruz, como requisito para obtenção do título de Mestre em Saúde Pública. Área de concentração: Território, Vigilância e Avaliação das Condições de Saúde; 2018.

31. Gil-Estevan MD, Solano-Ruiz MC. La aplicación del modelo de competencia cultural en la experiencia del cuidado en profesonales de Enfermería de Atención Primaria. Aten Primaria. 2017; 49(9):549-556. doi:10.1016/j.aprim.2016.10.013

32. Silva TA, Solano MdC, Siles J, de Freitas GF. Professional Identity of Nurse Manager in the Light of the Structural Dialectic Care Model. Aquichan. 2019; 19(3):e1935. doi: 10.5294/aqui.2019.19.3.5

33. Napier D (ed). Culture matters: using a cultural contexts of Health approach to enhance policy-making. Copenhagen, Denmark: World Health Organization; 2017

ISSN 1695-6141

( COPYRIGHT Servicio de Publicaciones - Universidad de Murcia 